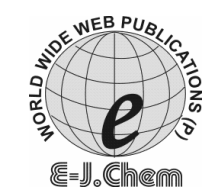

http://www.e-journals.net
ISSN: 0973-4945; CODEN ECJHAO

E-Journal of Chemistry

Vol. 5, No.4, pp. 782-791, October 2008

\title{
Saccaharum Cilliare Fiber Reinforced Polymer Composites
}

\author{
A.S. SINGHA* and VIJAY KUMAR THAKUR \\ Applied Chemistry Research Laboratory \\ Department of Chemistry \\ National Institute of Technology Hamirpur - 177005 \\ Himachal Pradesh, INDIA. \\ assingha@gmail.com,vijaythakurnit@gmail.com \\ Fax No. +91-1972 -223834; +91-1972-254120
}

Received 8 February 2008; Accepted 5 April 2008

\begin{abstract}
Renewable resources such as natural fibers in the field of fiber reinforced materials with their new range of applications represent an important basis in order to fulfill the ecological objective of creating eco-friendly materials. In views of enormous advantages a study on green composites using Saccaharum cilliare fiber as a reinforcing material and urea-formaldehyde (UF) as a novel matrix has been made. First of all urea-formaldehyde resin synthesized was reinforced with Saccaharum cilliare fiber. Reinforcement of the fiber was accomplished in three different forms particle (200 micron) reinforcement, short fiber $(3 \mathrm{~mm}$.) reinforcement and long fiber $(6 \mathrm{~mm})$ reinforcement. Present work reveals that mechanical properties such as: tensile strength, compressive strength and wear resistance of urea -formaldehyde resin (UF) increases to a significant extent when reinforced with Saccaharum cilliare fiber which is found in outsized amount in the Himalayan Region. These mechanical properties mainly depend upon the dimensions of the fiber used. Analysis of results shows that particle reinforcement is more effective as compared to short and long fiber reinforcement. Morphological and thermal studies of these composites have also been carried out.
\end{abstract}

Keywords: Polymer composites, Reinforcements, Optimization, Mechanical properties.

\section{Introduction}

Renewable resources in the field of fiber reinforced polymeric materials with their new range of applications represent an important basis in order to fulfill the ecological objective of creating eco-friendly material ${ }^{1-2}$. Because of their enormous properties such as low density, good mechanical performance, relatively low cost, low weight, less damage to 
processing equipment, good relative mechanical properties, unlimited availability and problem-free disposal, natural fibers offer a real alternative to the technical reinforcing fibers presently available ${ }^{3-4}$.An interesting environmentally friendly alternative for the use of synthetic fibers as reinforcement in engineering composites are lignocellulosic natural fibers such as flax, Grewia optiva, Pine needles, Hibiscus sabdariffa etc. ${ }^{5-7}$. Suitable matrix materials for natural-fiber reinforced polymers are polymer resin systems such as thermoplastics and thermosetting. Natural fibers like flax, Hibiscus sabdariffa, Pinus, Jute, Pineapple leaf fiber, Oil palm fiber have all been proved to be good reinforcements in thermoset and thermoplastic matrices ${ }^{8-13}$. Polymer composites can be used in many different forms ranging from structural composites in the construction industry to the high technology composites of the aerospace, space satellite and many other industries.

Saccharum cilliare fiber collected from higher reaches of Himalayan region has been found to contain $58.2 \%$ cellulose. Traditionally this fibrous material belonging to Himalayan region is being used by the people of the Himachal Pradesh for making low cost articles like ropes, bags, socks, boots, mats, etc. Literature survey has revealed that no work has been done on utilization of this raw fibrous material as reinforcing agents in polymer composites.

In this paper, synthesis of urea-formaldehyde resin and randomly oriented intimately mixed Saccharum cilliare fiber polymer composites by compression molding technique has been reported. The effect of fiber reinforcement particularly with $10 \%$ weight loading and effect of fiber dimension on the properties of the composites such as compressive strength, tensile strength, and wear resistance behaviour has been investigated. Fiber/matrix interaction was analyzed from the mechanical data, morphological (SEM) and thermal (TGA/DTA) studies.

\section{Experimental}

\section{Material and methods}

Urea, formaldehyde solution and sodium hydroxide kindly supplied by Qualigens Chemicals Ltd Company were used in this work. In the present research work we have used amino resin such as Urea-Formaldehyde (U-F) as Thermosetting Matrix Polymer.

Saccharum cilliare fiber was collected from higher reaches of Himalayan region in Himachal Pradesh. The fibers were washed thoroughly with distilled water and dried in an air oven at $80^{\circ} \mathrm{C}$ for $12 \mathrm{~h}$ and kept in a vacuum oven at $70^{\circ} \mathrm{C}$ for $3 \mathrm{~h}$ before preparation of composites. This fiber was used in three forms as shown below:

1. Particle reinforcement

Saccharum cilliare fibers were grinded to a powder and filtered through a sieve of pore size 200 microns.

2. Short- fiber reinforcement

Saccharum cilliare fibers were chopped into $3 \mathrm{~mm}$ size. These fibers were used as short fibers.

3. Long fiber reinforcement

Saccharum cilliare fibers were chopped into $6 \mathrm{~mm}$ size. These fibers were used as long fibers.

\section{Instrumentation}

Weights of the samples were taken on Shimadzu make electronic balance (LIBROR AEG220). Curing of different samples prepared was done on compression molding machine (SANTECH INDIA Ltd). Testing of samples for tensile and compressive strengths were 
done on ASTM made Computerized Universal Testing Machine (HOUNSFIELD H25KS), wear testing was done on Wear \& Friction Monitor (DUCOM- TR-20L). Morphological (SEM micrographs) and thermal studies (TGA/DTA, DTG) were carried out on (LEO 435VP) and on thermal analyzer (LINESIS, L81-11) respectively.

\section{Synthesis of urea-formaldehyde resin}

Urea-formaldehyde resin was synthesized by the standard method developed in our applied chemistry research laboratory. Urea and formaldehyde were taken in different molar ratio (1.0:1.0-1.0:3.0) by weight, in the reaction kettle. Both of these were mixed with the help of mechanical stirrer at a $\mathrm{pH}$ of 8.0. Initially temperature was maintained between $50-60^{\circ} \mathrm{C}$, for 2 hours. The chemical reaction is supposed to take place in two steps. First step involves the reaction between urea and formaldehyde to form methylol urea. Since urea is tetra functional, initial reaction may leads to the formation of tetra methylol derivative of urea (as shown in Figure 1).

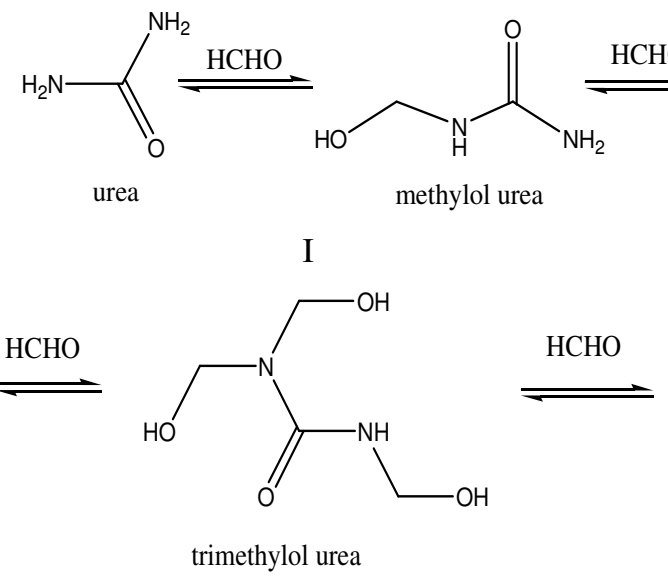

III<smiles>NC(=O)N(CO)CO</smiles>

II<smiles>O=C(N(CO)CO)N(CO)CO</smiles>

IV

Figure 1. Scheme of formation of methylol derivatives of urea

However slightly alkaline medium $(\mathrm{pH}$ of 7-8) control the reaction and favor the formation of dimethylol urea. Since these methylol urea are non adhesive in nature so condensation does not take place. However the condensation takes place in acidic medium and at higher temperature. Therefore the further reaction is carried out at $80-90^{\circ} \mathrm{C}$ and reaction speed is controlled by the acidity of the medium ( $\mathrm{pH} 5.5-6)$. Under acidic conditions methylol urea condenses by elimination of water between either of four steps (as shown in Figure 2).

As the reaction proceeds larger molecules with cross linked structures are formed. The condensation is intimately watched and controlled at the stages of fabrication since if the reaction is allowed to continue, cross linking leads to the gelatization of the resin. The reaction mixture was condensed at this temperature for 3-4h till the complete resinification. Then heating was stopped and the resin was cooled and samples were then cured at $120^{\circ} \mathrm{C}$ in compression molding machine under pressure $\left(400 \mathrm{~kg} / \mathrm{cm}^{2}\right)$ for initial 10 minutes. All the specimens after degasification were then post cured at $130^{\circ} \mathrm{C}$ for $12 \mathrm{~h}$. The cured samples were then subjected to various mechanical, thermal and morphological studies. 


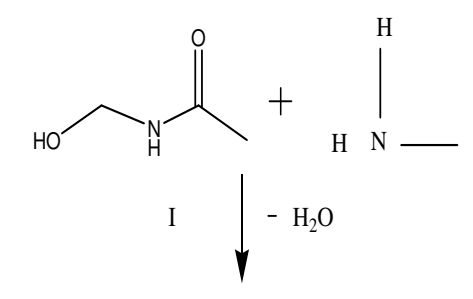<smiles>CCNC(C)=O</smiles>

III<smiles>CNC(=O)C(C)O</smiles>

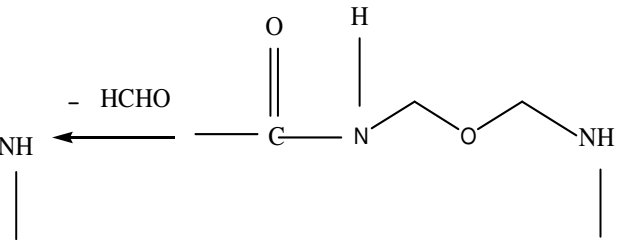

IV

Figure 2. Scheme of condensation of methylol urea by elimination

\section{Preparation of composites}

Randomly oriented fiber reinforced composites were prepared by taking different dimensions of the Saccharum cilliare fibers. Saccaharum Cilliare-urea resin matrix composite sheets of size $150 \times 150 \times 10.0 \mathrm{~mm}$ were prepared by a compression molding method as described elsewhere ${ }^{14}$.After thorough mixing of the resin and fibers, the mixture was poured in to the molds. The surfaces of molds were coated on the inside with oleic acid to avoid adhesion of the mixture and to allow easy removal of the composites. The molds were then closed and kept under pressure $\left(400 \mathrm{~kg} / \mathrm{cm}^{2}\right)$ and temperature 110 for initial 10 minutes. All the specimens after degasification were then post cured at $130^{\circ} \mathrm{C}$ for $12 \mathrm{~h}$.

\section{Mechanical characterization}

Composites thus prepared were cut into rectangular pieces of dimension $100 \times 10 \times 10 \mathrm{~mm}$ for the evaluation of tensile and compressive strength while of dimension $30 \times 10 \times 10 \mathrm{~mm}$ for wear resistance properties. The numbers of specimen used for the determination of mechanical properties were seven and the tests were conducted at ambient laboratory conditions.

\section{SEM analysis of samples}

Morphological analysis of UF resin and its respective composites was carried out by studying SEM micrographs. These SEM micrographs of the UF resin and composites show a clear cut difference in the morphology of the resin and its composite. These micrographs clearly show the difference between reinforced matrix and one which is not reinforced.

\section{Thermal Analysis of Samples}

Thermal analysis of materials gives us sufficient information about their thermal stability. Thermo gravimetric analysis (TGA) and differential thermal analysis (DTA) studies of samples were carried-out in air on a thermal analyzer at a heating rate of $10^{\circ} \mathrm{C} / \mathrm{min}$ the temperature ranging from $25^{\circ} \mathrm{C}$ to $1000^{\circ} \mathrm{C}$.

\section{Results and Discussion}

The investigation of mechanical properties of composites is one of the most important techniques in studying the behaviour of composite materials. It has been proved to be the most effective method to study the behaviour of the materials under various conditions of tension, compression, stress and phase composition of fiber composites and its role in 
determining the mechanical properties. Mechanical properties of fiber reinforced composites depend on the nature of the matrix material and the distribution and orientation of the reinforcing fibers, the nature of the fiber-matrix interfaces and of the interphase region. Even a small change in the physical nature of the fiber for a given matrix may result in prominent changes in the overall mechanical properties of composites. It is well known fact that different degrees of reinforcement effects are achieved by the addition of hydrophilic fibers to different polymers. This may be due to the different adhesion strength between matrix and fibers. The adhesion is usually the strongest in polar polymers capable of forming hydrogen bonds with hydroxyl groups available on the natural fiber surface.

\section{Mechanical properties of composites}

Preparation of urea-formaldehyde (UF) Resin based composites has been done by employing the optimized ratio (1:2.5) of UF resin. Optimization of UF resin has been done by evaluating mechanical properties such as tensile strength, compressive strength and wear resistance etc. From Figures (3-4) it is clear that U-F resin in the ratio 1.0: 2.5 bears load of 128.125 NF with an extension of $1.84 \mathrm{~mm}$ under tension, a load of $991 \mathrm{~N}$ at a compression of $3.51 \mathrm{~mm}$ under compression which are greater than the results of other ratios. Further the wear rate of samples of ratio 1.0:2.5 was less as compared to any other samples (Figure 5).

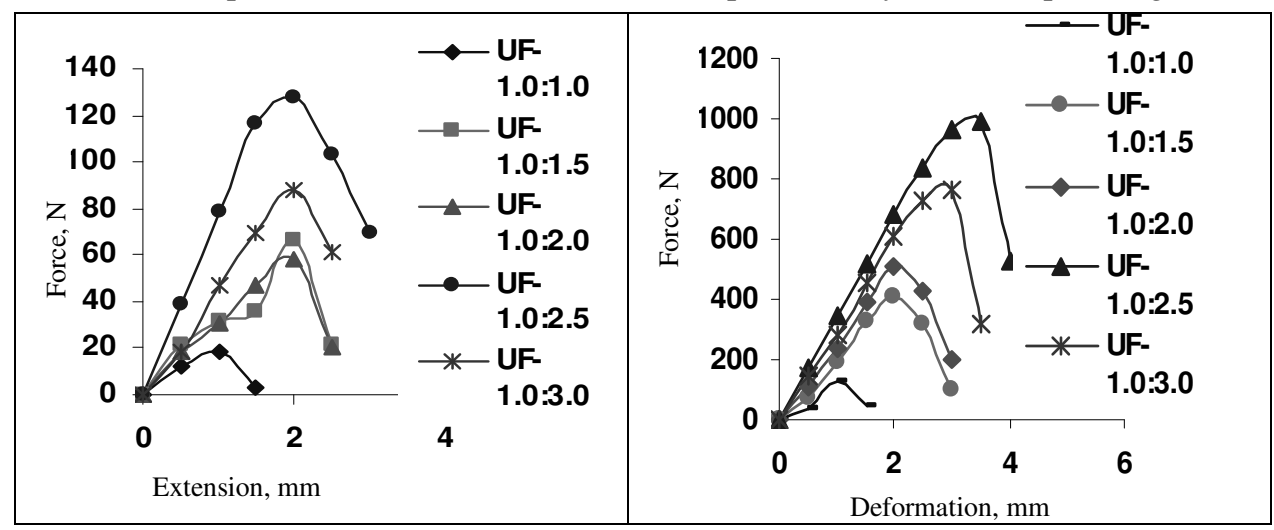

Figure 3. Tensile strength of UF Resin

Figure 4. Compressive strength of UF Resin

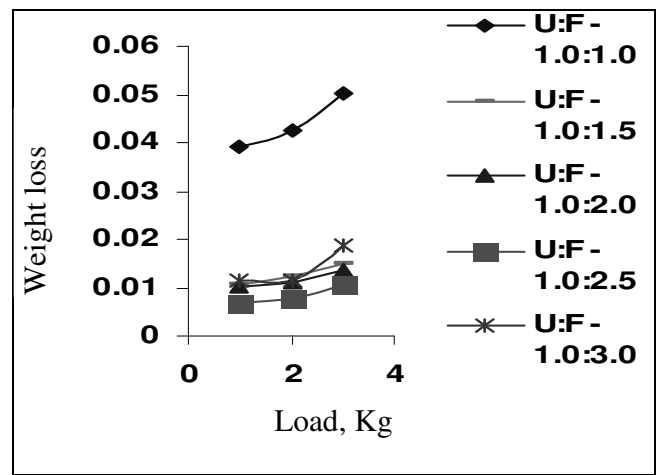

Figure 5. Wear resistance of UF resin Rnf 


\section{Compressive strength}

Compressive strength of UF matrix has been found to increase on reinforcement with Saccharum cilliare fiber. It has been found that on particle reinforcement compressive strength increase to a much greater extent as compared to other reinforcement (short and long fiber reinforcement). From Figures (6-8) it is clear that (i) Particle reinforced composite bear a load of $1745 \mathrm{~N}$ with compression of $3.61 \mathrm{~mm}$ (ii) Short fiber reinforced composite bear a load of 1570.0 with compression of $3.71 \mathrm{~mm}$ (iii) Long fiber reinforced composite bear a load of $1480.0 \mathrm{~N}$ with compression of $3.73 \mathrm{~mm}$.

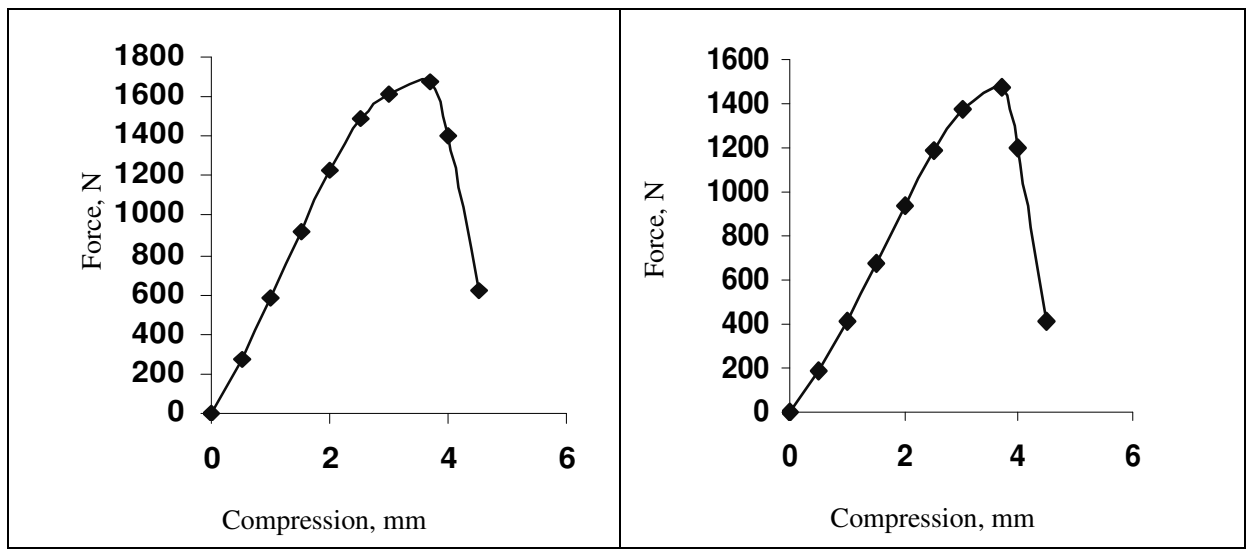

Figure 6. Compressive strength of P- Figure 7. Compressive strength of SFcomposite

Rnf composite

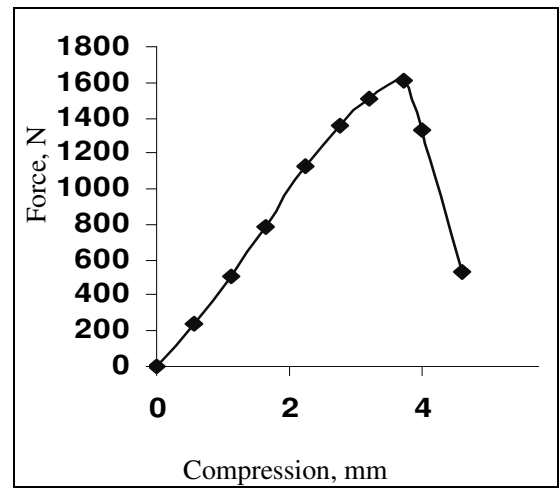

Figure 8. Compressive strength of LF- Rnf composite

\section{Tensile strength}

It has been observed that composites with particle reinforcement showed more tensile strength which was followed by short fiber and continuous fiber reinforced composites.

From Figures (9-11) it is clear that (i) Particle reinforced composite bear a load of $221.44 \mathrm{~N}$ with an extension of $1.94 \mathrm{~mm}$ (ii) Short fiber reinforced composite bear a load of $200.0 \mathrm{~N}$ with extension of $1.98 \mathrm{~mm}$ (iii) Long fiber reinforced composite bear a load of $193.0 \mathrm{~N}$ with extension of $2.0 \mathrm{~mm}$. 


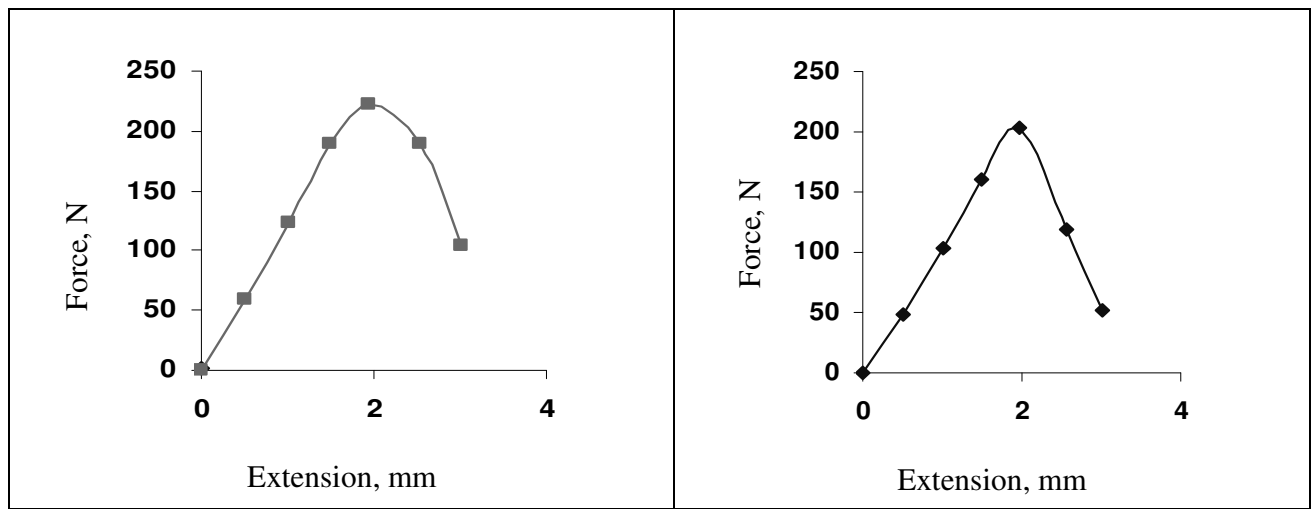

Figure .9. Tensile strength of P- Rnf composite

Figure 10. Tensile strength of SF- Rnf composite

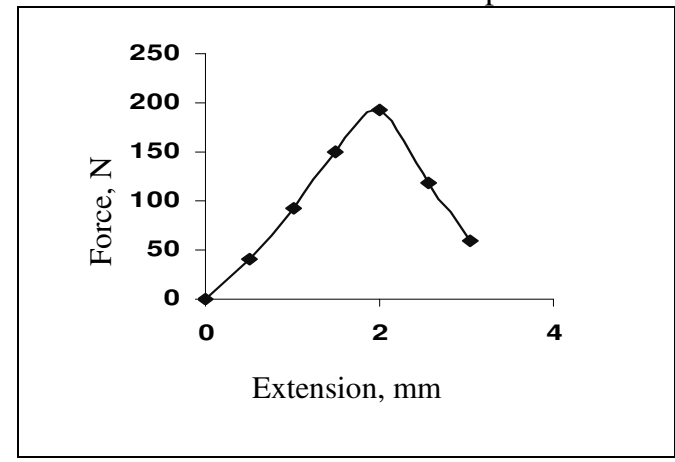

Figure 11. Tensile strength of LF- Rnf composite

\section{Wear Resistance}

As evident from Figures (12-14) that wear rate of UF matrix decreases appreciably as reinforcement with Saccharum cilliare fiber. Further with decrease in dimension of the fiber the wear resistance increases.

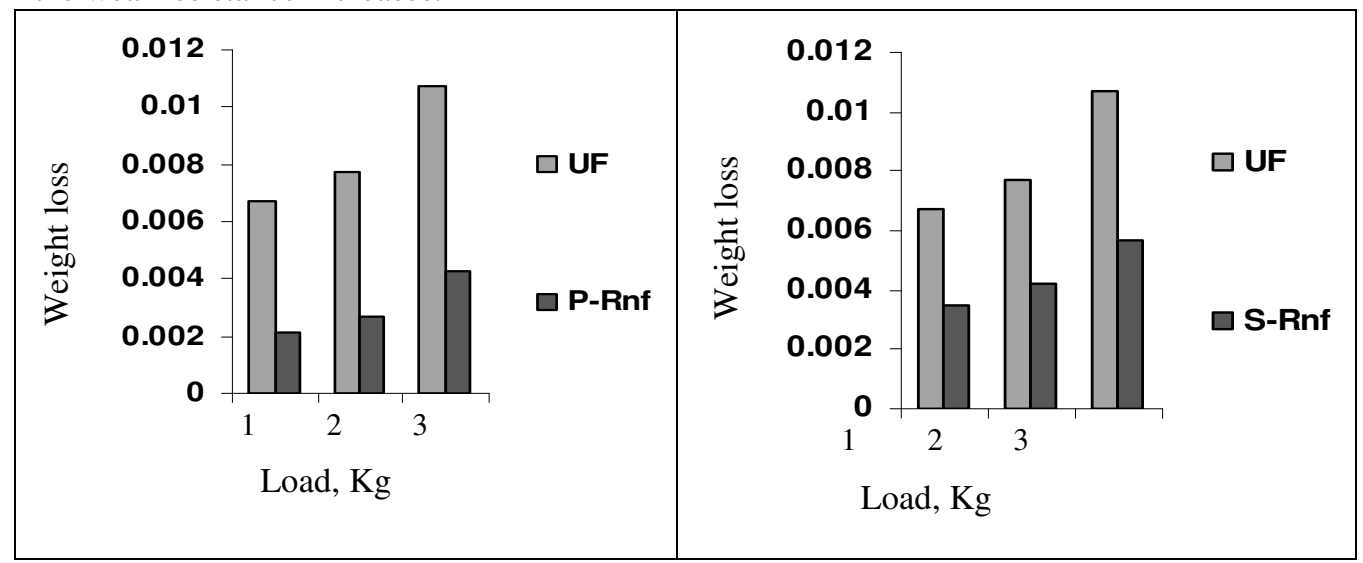

Figure 12. Wear resistance of P-Rnf composite
Figure 13.Wear resistance of SF-Rnf composite 


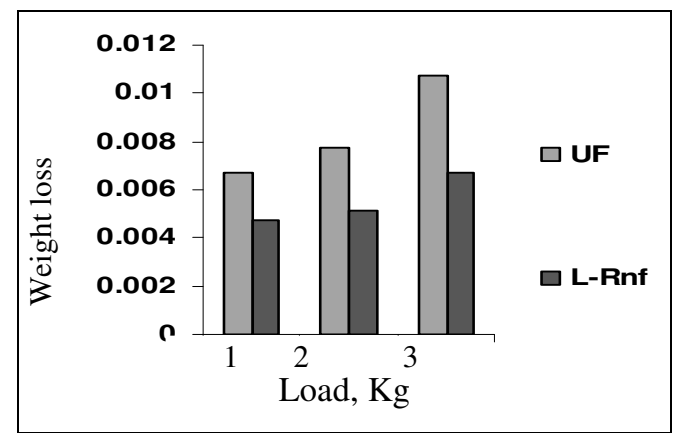

Figure 14.Wear resistance of SF-Rnf composite

From these results it is clear that mechanical properties of the matrix increases to a greater extent when reinforced with natural fibers. The major reason for this is the bonding between the matrix and the fibers. The chemical bonding accounts for the adhesion between amino resin (urea formaldehyde) and the natural fibrous material. The higher bond strength obtained for amino resin matrix is due to the possible reaction between the methylol groups of the resin with the hydroxyl group of cellulose.

Further from these results it is clear that particle reinforcement is more effective than of short and long fiber reinforcements. This may be due to larger surface area and more fiber/matrix interaction in case of particle reinforced as compared to short and long fiber reinforced composites.

\section{Thermal behaviour of $U-F$ resin and its composites}

Thermogravimetric analysis (TGA) of materials such as raw fiber, polymeric resin and composites was studied as a function of $\%$ weight loss with the increase in temperature (Table 1). Results indicate that the presence of cellulose fibers affects the degradation process of the composites. These studies are further supported by differential thermal analysis (DTA), the results are shown in Table 2. These results are consistent with results reported earlier.

Table 1. Thermogravimetric analysis of UF, SC and P-Rnf-UF composites where $U F=$ Urea formaldehyde resin; $S C=$ Saccaharum Cilliare

\begin{tabular}{ccccccc}
\hline S. No & Sample Code & IDT, ${ }^{0} \mathrm{C}$ & $\%$ wt. loss & FDT, ${ }^{0} \mathrm{C}$ & $\%$ wt. loss & Final Residue, $\%$ \\
\hline 1. & SC & 212 & 12.45 & 471 & 67.44 & 32.66 \\
2. & U-F Resin & 238 & 22.48 & 993 & 87.51 & 12.49 \\
3. & P-rnf-UF & 231 & 25.51 & 785 & 73.53 & 26.47 \\
\hline \multicolumn{7}{c}{$P$-rnf $=$ Particle reinforced composite }
\end{tabular}

Table 2. Differential thermal analysis of UF, SC and P-Rnf-UF composites

\begin{tabular}{lll}
\hline S. No. & Sample Code & Exothermic/Endothermic peaks ${ }^{0} \mathrm{C}, \mu \mathrm{V}$ \\
\hline 1. & SC & $326.0(81) ; 430.0(203)$ \\
2. & U-F Resin & $179[6.8] ; 253[5.7] ; 271[27.9] ; 545[9.4] ; 725[-23.0]$ \\
3. & P-Rnf-UF & $75[2.4] ; 263[21]$ \\
\hline Where & UF = Urea formaldehyde resin; SC = Saccaharum cilliare \\
\multicolumn{2}{l}{ P-rnf= Particle reinforced composite }
\end{tabular}




\section{Morphological study of composites}

Morphological results (Figure15-18) clearly show that there is proper intimate mixing of fiber with the resin in the composites thus synthesized.

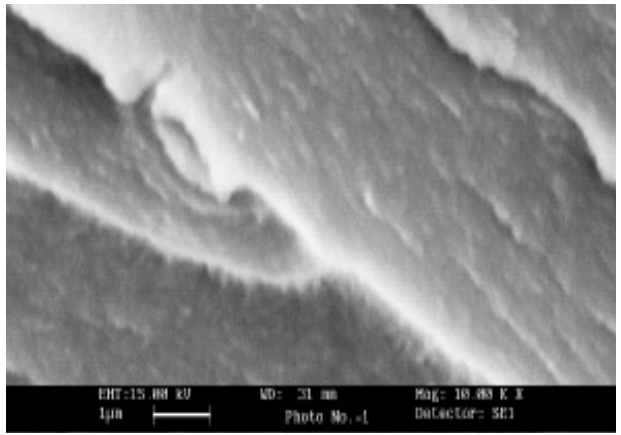

Figure 15. SEM of UF Resin

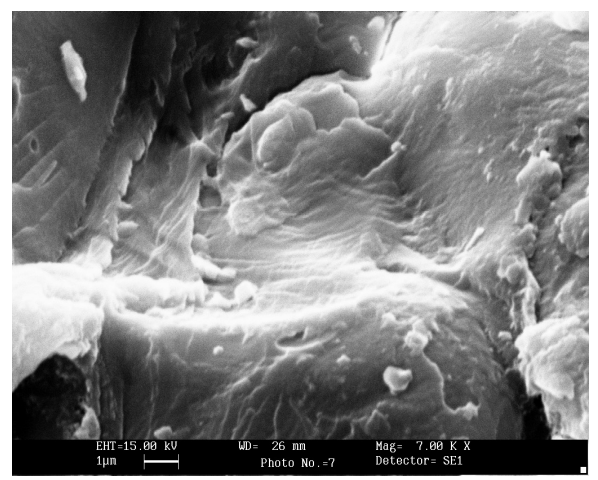

Figure 17. SEM of SF-Rnf composite

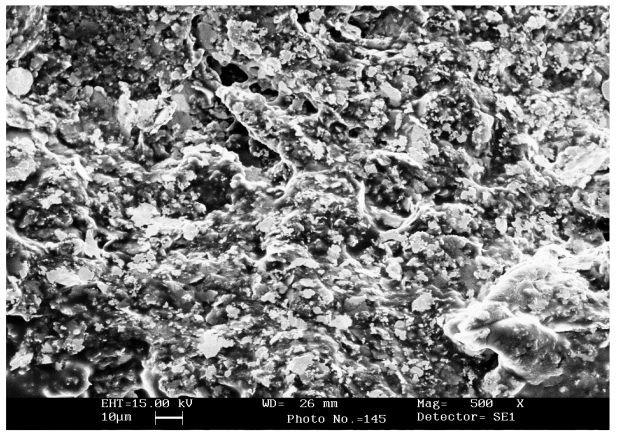

Figure 16. SEM of P-Rnf composite

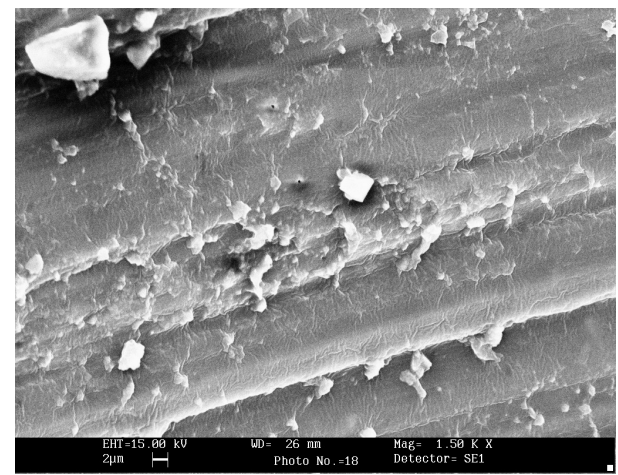

Figure 18. SEM of SF-Rnf composite

\section{Conclusions}

Various test methods were adapted for complete mechanical characterization of natural fiber reinforced composites. In case of mechanical behaviour particle reinforcement of the UF resin has been found to be more effective as compared to short and long fiber reinforcement. This could be due to more matrix- particle interfacial interaction as compared to short fiber/ continuous fiber-matrix interfacial interaction. These results suggest that Saccaharum cilliare fiber is a potential candidate for reinforcement in polymer composites.

\section{Acknowledgement}

We express our sincere thanks to Director, National Institute of Technology Hamirpur for providing the basic laboratory facilities.

\section{References}

1. Kaith B S , Singha A S, Sanjeev Kumar and Susheel Kalia, Int J Polym Mater., 2008, 57(1), $54-72$.

2 Bledzki A K and Gassan J, Prog Polym Sci., 1999, 24, 221-274. 
3 Singha A S, Kaith B S and Sarwade B D, Hungarian Journal of Industrial Chemistry, 2002, 30, 289-293.

4 Panthapulakkal S, Zereshkian A and Sain M, Bioresource Technol., 2006, 97, 265-72.

5 Kaith B S, Singha A S, Susheel Kumar and Misra B N, J Polym Mater., 2005, 22, 425- 432 .

6 Bledzki A K, Reihmane S and Gassan J, J Appl Polym Sci., 1996, 59(8),1329-1336.

$7 \quad$ Singh B, Verma A and Gupta M, J Appl Polym Sci., 1998, 70, 1847-1858.

8 Gatenholm P, Bertilsson H and Mathiasson A, J Appl Polym Sci., 1993, 49(2),197-208.

9 Maldas D and Kokta B V, Trends Polym Sci., 1993, 1(6),174-8

10 Bledzki A K, Reihmane S and Gassan J, Polym Plast Technol Eng., 1998, 37(4), 451-68.

11 Nabi Saheb D and Jog J P, Adv Polym Technol, 1999, 18(4), 351-63.

12 Saheb Nabi and Jog J P, Advances in Polymer Technology, 1999,18(4) 351-363.

13 Maya J, Bejoy F, Sabu T and Varughese K T, Polymer Composites, 2006, 27(6), 671-680.

14 Singha A S, Kumar S and Kaith B S, Int. J. Plast. Tech., 2005, 9, 427. 


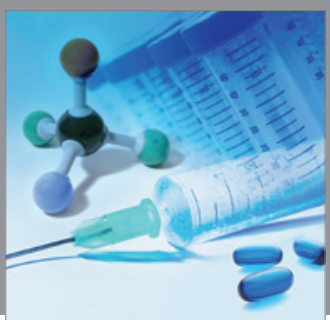

International Journal of

Medicinal Chemistry

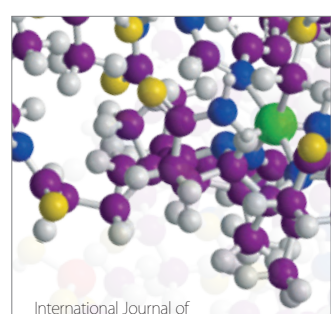

Carbohydrate Chemistry

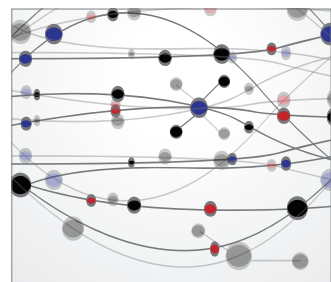

The Scientific World Journal
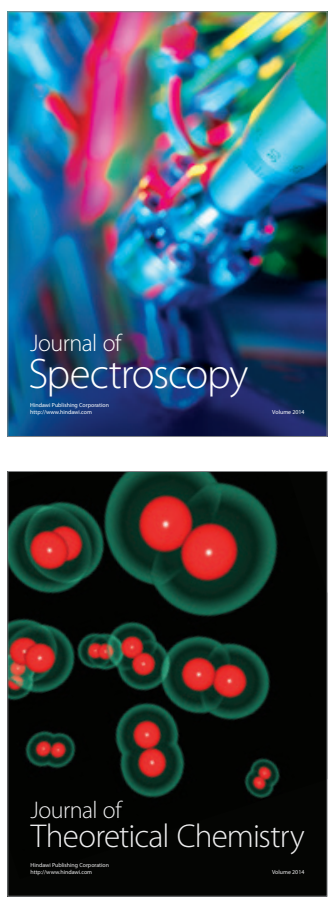
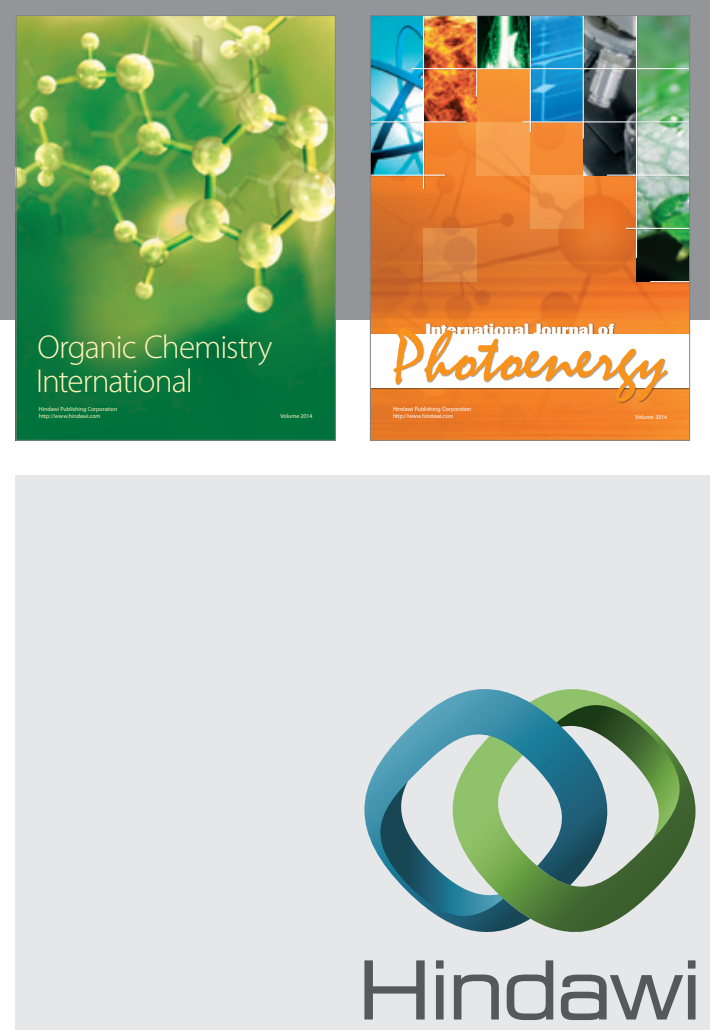

Submit your manuscripts at

http://www.hindawi.com
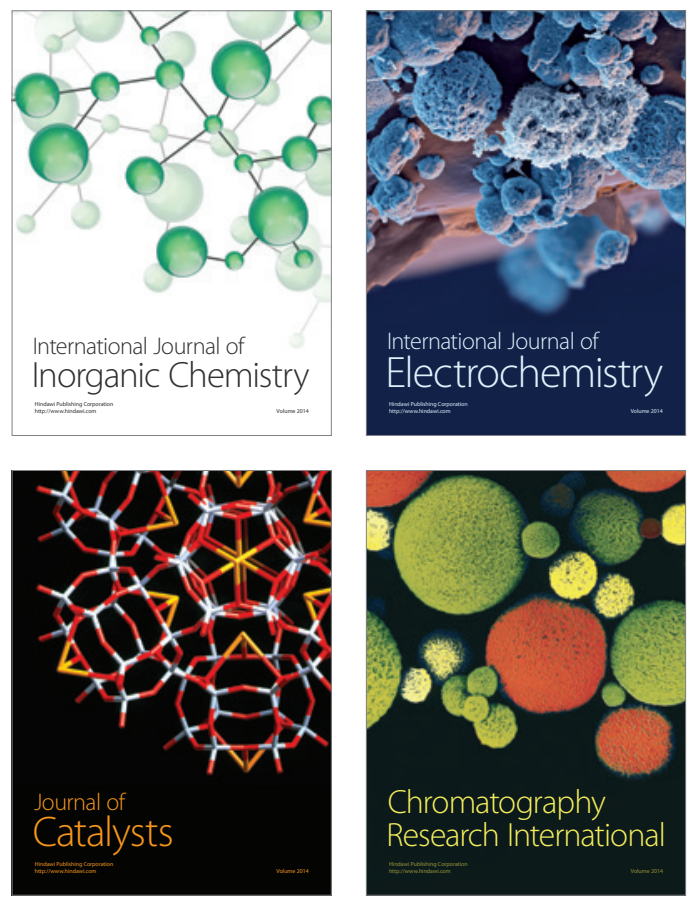
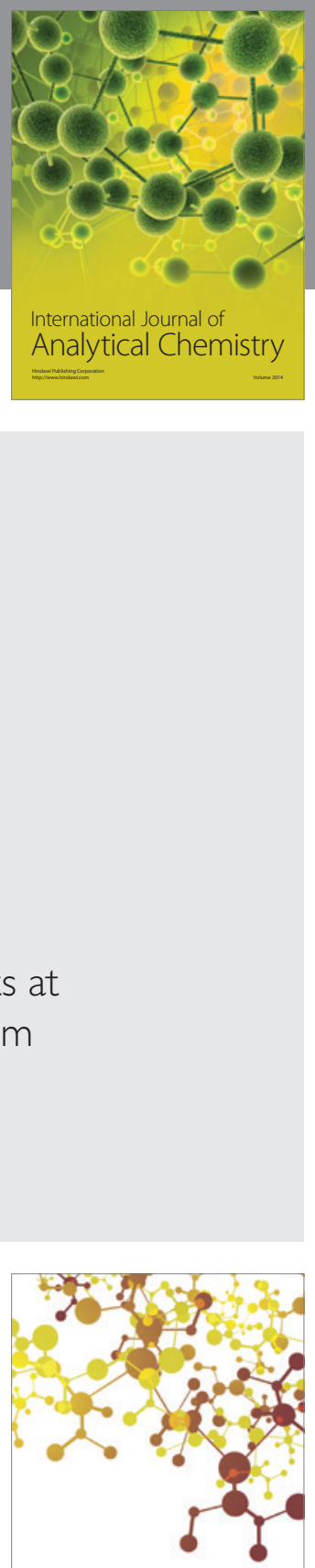

Journal of

Applied Chemistry
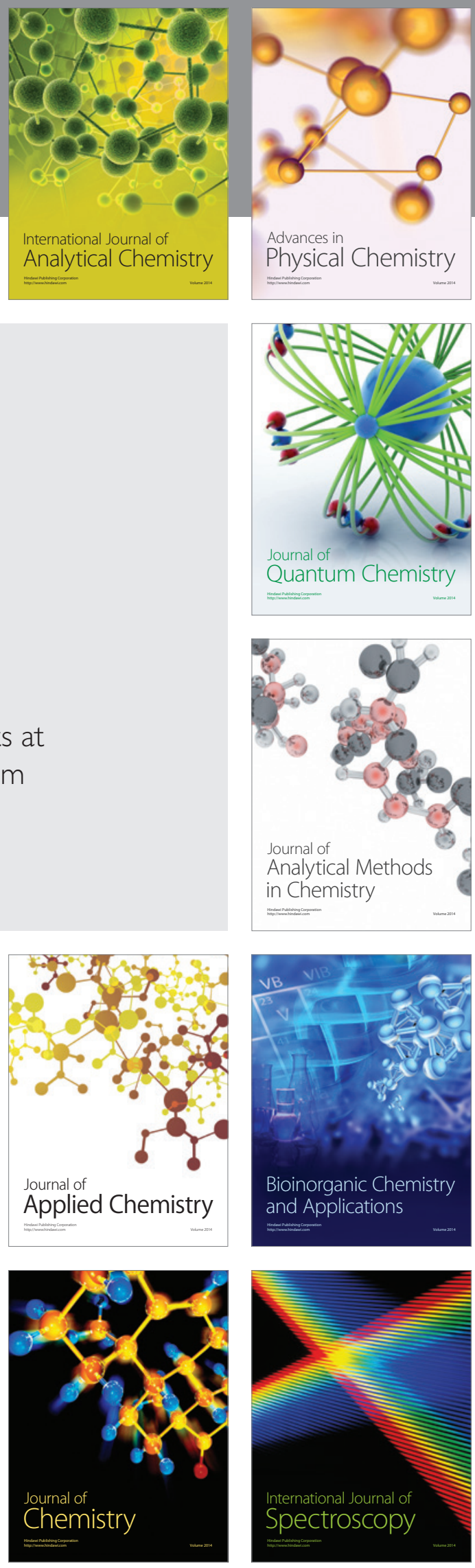